\title{
1 COMMIT: Consideration of metabolite leakage and community \\ 2 composition improves microbial community models
}

3 Philipp Wendering ${ }^{1}$, Zoran Nikoloski ${ }^{1,2^{*}}$

$4{ }^{1}$ Bioinformatics, Institute of Biochemistry and Biology, University of Potsdam, 14476 Potsdam,

5 Germany

$6 \quad 2$ Systems Biology and Mathematical Modeling, Max Planck Institute of Molecular Plant

7 Physiology, 14476 Potsdam, Germany

8 * Email: nikoloski@mpimp-golm.mpg.de, Address: Karl-Liebknecht-Str. 24-25, 14476 Potsdam-

9 Golm, Tel: +493319776305

10

11

12 Composition and functions of microbial communities affect important traits in diverse hosts, from

\section{Abstract} crops to humans. Yet, mechanistic understanding of how metabolism of individual microbes is affected by the community composition and metabolite leakage is lacking. Here, we first show that the consensus of automatically generated metabolic models improves the quality of the draft models, measured by the genomic evidence for considered enzymatic reactions. We then devise an approach for gap filling, termed COMMIT, that considers exchangeable metabolites based on their permeability and the composition of the community. By applying COMMIT with two soil communities from the Arabidopsis thaliana culture collection, we could significantly reduce the gapfilling solution in comparison to filling gaps in individual models. Inspection of the metabolic interactions in the soil communities allows us to identify microbes with community roles of helpers and beneficiaries. Therefore, COMMIT offers a versatile automated solution for large-scale modelling of microbial communities for diverse biotechnological applications.

\section{Main Text}

\section{Introduction}

Microbial communities have been extensively studied due to their importance in ecology $(1,2)$, human health $(3,4)$, and biotechnological applications (5). It has also been suggested that microorganisms have particular roles in a community; for instance, the Black Queen (BQ) hypothesis (6) suggests the existence of $B Q$ functions, such as production of membrane-permeable products, that are essential for members termed helpers, but unavoidably available to other community members, termed beneficiaries. These roles are achieved by active transport and/or leakage of diverse metabolites, including biomass precursors (7).

Constraint-based modelling of genome-scale metabolic networks provides the means to in silico analyse microbial community interactions (8-12). The existing metabolic reconstruction 
37 approaches (13-19) rely on linking genome annotation to enzymatic reactions from various 38 databases (19-23). A structural comparison of the metabolic models resulting from these 39 approaches showed that the portions of shared reaction, metabolite, and gene sets are rather 40 moderate (24). Hence, a consensus model could provide a means to combine the advantages of 41 the existing approaches $(25,26)$.

42 However, a consensus model is not guaranteed to be functional (i.e. to simulate growth), as 43 knowledge gaps may occur in all of the underlying (draft) models. To address this issue, several 44 algorithms for gap filling, applicable to single models, have been proposed; however, they are not 45 feasible for gap filling models of large communities due to the sheer amount of computational 46 resources required $(27,28)$. Since members of microbial communities are often dependent on each 47 other, gap-filling solutions of the community members must also be put into context of the 48 community in which the organisms co-exist. In addition, the usage of gap-filled solutions and 49 exudates from other community members may further reduce the overall number of added 50 reactions to fill in the gaps in the metabolic model for the community. Existing computational efforts 51 have shown that the gap-filling medium as well as the order in which gap-filling is applied plays a 52 very important role in the reconstructed model (29).

53 Further, the problem of finding metabolic interactions within microbial communities using constraint54 based methods has been addressed by multiple studies (8, 10, 30-34). However, most of these 55 approaches are not suited for analysis of large microbial communities, comprising more than 10 56 microorganisms, due to the sheer size of the arising constraint-based problems. Importantly, the 57 fully automated approaches rely solely on transport reactions in the model, and neglect permeability 58 to define the set of exchangeable metabolites.

59 Here, we describe COMMIT, a constraint-based approach that respects the composition of the 60 microbial community and metabolite leakage in the process of gap filling the consensus metabolic 61 models of the respective community members. We then apply COMMIT to reconstruct high-quality 62 metabolic models based for operational taxonomic units (OTUs) from the Arabidopsis thaliana 63 microbial culture collections, At-SPHERE, for two different soil community compositions $(35,36)$.

64 Altogether, our results demonstrate that the consensus approach in combination with the gap filling 65 approach that respects the community composition renders COMMIT a valuable addition to the 
66 approaches for fully automated reconstruction of large-scale metabolic models of microbial 67 communities.

\section{Results}

Reconstructed draft genome-scale metabolic models for 432 OTUs in At-SPHERE show substantial structural differences

74 We used the high-quality genomes for 432 OTUs available from At-SPHERE (37) to reconstruct 75 draft genome-scale metabolic models. To this end, we applied four widely-used, fully automated approaches for metabolic network reconstruction relying on few parameters, namely: KBase (19), CarveMe (14), RAVEN 2.0 (17), and AuReMe/ Pathway Tools (15, 16) (for reviews, see (24, 38)). We then converted the draft models reconstructed by the four approaches to a common format by multiple technical adaptations (see Methods). To compare the structure of the models after the conversion, we employed eight distance measures, including: the Jaccard distance based on the sets of metabolites, reactions, E.C. numbers, and dead-end metabolites, the number of dead-end metabolites, the rank correlation of E.C. number occurrence, usage of cofactors, the SVD distance of the stoichiometric matrices, and the Jaccard distance of gene identifiers (see Supplementary Methods). We then combined the resulting distance matrices in a compromise distance matrix for each OTU; to facilitate a global comparison of the different approaches, we also calculated the compromise distance matrix over all OTUs (see Methods, Figure 1A). The structural comparison revealed substantial differences across the draft genome-scale metabolic models generated by the four approaches (Figure 1A). In the compromise distance matrix obtained from the eight distance measures across all OTUs, the draft models showed an average distance of 0.64 to each other, ranging from 0.54 to 0.72 (with 1 denoting the largest difference). Regarding the gene identifiers, the models reconstructed using KBase, CarveMe, and RAVEN 2.0 were more similar to each other in comparison to the models resulting from AuReMe/Pathway Tools.

94 To determine whether or not the utilized distance measures are biologically relevant, we next calculated the correlation between the distance matrices and sequence dissimilarity of the $16 \mathrm{~S}$ rRNA sequences. We found that the Jaccard distances corresponded to the generated phylogeny, with significant correlations ranging from 0.63 to 0.75 with an average of $0.70(p<0.001)$ over the 432 OTUs (Figure 1B). We also observed moderate but significant correlations of the SVD distance and the rank correlation of cofactor usage with sequence dissimilarity $(\bar{\rho}=0.25, p<0.001)$. In contrast, the occurrence of dead-end metabolites showed a very low correlation with sequence dissimilarity $(\bar{\rho}=0.04)$. These findings indicated that the utilized distance measures to quantify the 
103 supporting our result that the models obtained by some of the utilized approaches are strikingly

104 different (Figure 1A).

\section{Consensus metabolic models show high organism specificity}

107 It has been demonstrated that the integration of multiple metabolic models into a consensus model 108 leads to a reduced number of blocked reactions due to the complementarity of their information 109 content $(25,39,40)$. Since we observed that the draft models generated with the four reconstruction 110 approaches differed in their underlying genome annotation and downstream reaction and 111 metabolite sets (Figure 1A), we hypothesized that there would be an overall increase in model 112 quality (assessed by the decrease in the number of gaps) of a corresponding consensus model. 113 The consensus generation consisted of matching metabolite, reaction, and gene identifiers. Since 114 the metabolites in the MetaNetX database are already structurally matched between various 115 databases, duplicate metabolites could be removed from the consensus by only considering their 116 identifiers. We employed cosine similarity to identify reactions of similar stoichiometry that may 117 have opposite directions, lack protons or whose coefficients differ by a factor. Further, we compared 118 mass-balance, reversibility, direction, and protonation. Previously published approaches as 119 COMMGEN (40) or MetaMerge (25) were not applicable since they do not support the current 120 MNXref format used in the MetaNetX database or are no longer maintained.

121 We found that the consensus model is considerably smaller than the mere sum for reactions, 122 metabolites, and genes contained in the underlying draft models (Figure 1C). Further, the 123 proportions of reactions, metabolites, and genes were not uniform across the draft models obtained 124 from the different reconstruction approaches. The RAVEN 2.0 reconstructions consistently showed 125 larger numbers of reactions, metabolites, and genes compared to all other methods, with 126 AuReMelPathway Tools draft models exhibiting the lowest sums of these properties (Figure 1C). 127 As a result, the draft models from RAVEN 2.0 exhibited the smallest overall distance (0.37) to the 128 consensus model, while the draft models obtained from the other tools showed an average distance 129 of 0.59 to the consensus (Figure $1 \mathrm{~A}$ ). The Jaccard distances of gene identifiers to the consensus 130 model ranged between 0.31 for RAVEN 2.0 reconstructions and 0.87 for AuReMe models.

131 To show the organism-specificity of the resulting consensus model, we next assessed the similarity 132 of the consensus to selected reference models for OTUs that were resolved down to species 133 classification, namely Bacillus megaterium (iMZ1055) and Methylobacterium extorquens (iRP911). 134 To this end, we assessed the manually-curated models for these two species $(41,42)$ by first 135 translating their identifiers into the MNXref namespace (whenever possible), facilitating comparison 136 of reactions, metabolites, and E.C. numbers. Every consensus model was compared to each of the 137 two curated models to determine the number of true positives, false positives, and false negatives 138 and to calculate sensitivity and precision (see Methods). Both values were scaled by the sequence 
139 similarity of an OTU's 16S rRNA sequence to the sequence of the reference species. Despite the

140 low translation efficiency, we found that the consensus models of OTUs of the same species were

141 more similar to the curated models than models of OTUs that share only the genus or model of

142 even less-related OTUs (Figure 1D). Notably, most of the models for OTUs with only matching

143 genus exhibited higher precision for $M$. extorquens than the ones from the same species. Moreover,

144 the consensus models showed higher values for scaled sensitivity compared to the individual draft

145 models (Figure S1). In contrast, the values for the scaled precision of the consensus models were

146 smaller than those of the draft models. This can be explained by the overlap of the draft models

147 with respect to the sets of features (i.e. metabolites, reactions, and E.C. numbers): The number of

148 the non-overlapping features (i.e. false positives) increases more than the number of features

149 matching with the reference models (i.e. true positives) during the consensus generation. Hence,

150 the precision is lower for the consensus as the ratio of true positives to false positives is shifted.

151 Yet, across all draft models, except for AuReMelPathway Tools, models of the same species and

152 genus were more similar to each other than models of phylogenetically more distant OTUs.

153 Therefore, we concluded that the consensus of metabolic models shows a higher organism

154 specificity as well as a higher quality than the draft models obtained from the individual approaches.

156 COMMIT provides gap-filling that respect the community composition and metabolite 157 leakage

158 Constraint-based analysis of microbial communities is based on assembling functional metabolic 159 models for members of the community that can, in turn, be used to simulate microbial growth.

160 However, obtaining a functional metabolic model from a draft model entails adding reactions to an 161 incomplete metabolic network to enable simulation of growth $(43,44)$; often the inserted reactions 162 are without genomic support.

163 The existing gap-filling methods are applied with models of individual species and do not consider 164 the innate dependence between the community members $(6,9,45)$. In contrast, our approach, 165 termed COMMIT, aims to identify a minimal gap-filling solution that respects the composition of the 166 microbial community. To this end, COMMIT explores a specified number of random orderings of 167 the community members. Draft models for each member of the community are then gap-filled 168 following a given random ordering of the community members. COMMIT relies on the 169 FastGapFilling algorithm, which optimizes a weighted sum of the fluxes through the biomass and 170 additional candidate reactions by using linear programming (LP) formulation (see Methods section 171 for more detail).

172 More specifically, COMMIT starts with a minimal medium; exported metabolites from gap-filled 173 models are then determined and used to enlarge the medium, thereby shaping the overall gap174 filling solution. The random orderings are compared with respect to four criteria, including: (i) the 
175 number of added reactions, (ii) dependence of the first member on exported metabolites of 176 subsequent models, (iii) number of exchanged metabolites, and (iv) the sum of biomass fluxes of

177 all community members (see Methods). We determined exchangeable metabolites after gap-filling

178 each of the draft models following the given random ordering of community members. A metabolite

179 is considered exchangeable if its export does not decrease the flux through the biomass reaction

180 by more than a pre-specified factor (e.g. 10\%). We rely on using a threshold (of $10 \%$ ) because an

181 overly altruistic behaviour of single community members is not considered biologically relevant.

182 The set of exported metabolites is in turn made available to the subsequent models by allowing a 183 lower cost for their import, thus enlarging the initial gap-filling medium (Figure 2A). Assuming that 184 the community members depend on each other (6), we hypothesized that the enlarged medium is 185 expected to reduce the added reaction sets of subsequent members of the community. Further, 186 the existence of an optimal ordering is likely since gap-filling solutions do not only depend on the 187 algorithm and candidate reactions employed, but also on the underlying medium (29). Given an 188 ordering of microorganisms, the solution for the first model in the ordering is obtained by using its 189 auxotrophic medium used in KBase (13). This strategy was employed to prevent an unrealistically 190 high number of added reactions for the first model assuming the included compounds, most 191 importantly essential amino acids, are likely produced by other community members. Altogether, 192 the gap-filling procedure is conditional on the community composition, as it takes metabolic 193 capabilities of all community members into consideration.

194 In addition to the medium, the choice of candidate reactions affects the set of reactions that are 195 added to close gaps in biological pathways (46). In COMMIT, the objective of the gap-filling LP 196 includes specific costs for different reaction types (e.g. transport reactions for highly-permeable 197 metabolites and reactions with sequence evidence). To predict metabolite permeability, we 198 obtained molecular properties from PubChem (47) (see Methods section for details). Altogether, 199 six parameters were used for permeability prediction based on Lipinski's rule (48, 49), resulting in 200 reduced costs for 2766 out of 4520 transport reactions in the gap-filling database. Moreover, 201 reactions assigned to enzymes with sequence evidence in the respective genome were assigned 202 a lower cost (see Methods).

204 COMMIT reconstructs high-quality soil microbial communities based on consensus models 205 from At-SPHERE

206 We applied COMMIT to two soil microbial communities whose composition was determined by 207 comparison of $16 S$ rRNA sequences from environmental samples with those from the cross208 reference OTUs in At-SPHERE (37). This resulted in 20 and 24 recovered soil OTUs from two 209 respective studies which, referred to as "Bulgarelli" (36) and "Schlaeppi" (35). The analysis of OTU 210 abundances revealed the existence of high- and low-abundant OTUs in both experiments (Table 
211 S1, S2). The draft metabolic models for the members of the two communities were gap-filled by

212 applying COMMIT with 100 iterations, corresponding to different random orderings, and an initial

213 gap-filling medium containing only D-glucose as a carbon source (Table S3). The explored

214 orderings were compared with respect to the number of added reactions, biomass fluxes, and

215 number of exchanged metabolites, after scaling by their values in the optimal solutions (using the

216 four abovementioned criteria).

217 Since solutions with the smallest number of added reactions across all iterations are generally

218 favoured, all suboptimal solutions have a larger number of added reactions (Figure 3). Moreover,

219 for both communities, we observed a shift in the number of exchanged metabolites when comparing

220 the optimal with the suboptimal solutions from the other random orderings. While most of the

221 suboptimal solutions exhibited a larger number of exchanged metabolites in the full consensus

222 (Figure $3 \mathrm{~A}, \mathrm{~B}$ ), the peak shifts towards most solutions having a smaller number of exchanged

223 metabolites in the consensus without CarveMe models (Figure 3C,D) and consensus only from

224 KBase draft models (Figure 3E,F). This observation was likely due to the increasing importance of

225 integrating more exchange reactions for metabolites provided by other community members to

226 reduce the number of added reactions, when the number of merged models is lower. In contrast,

227 such a pronounced trend could not be observed for the sums of predicted growth rates.

228 Nevertheless, we observed that the distribution of scaled differences for the sum of biomass fluxes

229 is similar to the distribution of exchanged metabolites for most of the simulations, suggesting a

230 dependence between these two criteria.

231 We next used the memote test suite to assess the quality of the resulting functional models. The 232 generated models of the Schlaeppi community exhibited an average score of $35 \%$ (Table S4). A

233 strong reduction in the memote score was expected due to missing metabolite, reaction, and gene

234 annotation by databases other than MetaNetX. Nevertheless, the generated models showed an

235 average consistency of $44 \%$. Importantly, the average fraction of reactions with associated GPR

236 rules in the consensus was $90 \%$, since reactions with genomic evidence were preferred during both

237 the merging and gap-filling procedures. Therefore, the resulting models showed very similar

238 characteristics to the previously used references models. In comparison, the reference model for

239 B. megaterium, iMZ1055, was scored with $25 \%$ and $55 \%$ consistency.

\section{COMMIT shapes and significantly reduces the gap-filling solution}

242 The observed differences between different random orderings of community members indicated

243 that there exists an optimal solution that minimizes the number of added reactions for the particular

244 community composition. Next, we were interested to assess the differences between the gap filling

245 solutions for individual models with and without respecting the community composition. We found

246 that COMMIT decreased the number of added reactions compared to the individual gap filling of 
247 the models using the same algorithm without allowing for metabolite exchange between the

248 members (Figures 4A, S2). Despite the low number of added reactions for the consensus model,

249 we observed a significant decrease in the gap-filling solution size with COMMIT in comparison to

250 the classical gap filling approach (Figure 4A).

251 To show that the set of added reactions is not only smaller but also differs in its composition, we 252 performed $\mathrm{K}$-means $(K=3)$ clustering with the matrix of Jaccard similarities of the added reaction

253 sets for the full consensus, consensus without CarveMe, and KBase draft models gap-filled either

254 with or without considering the community composition (Figures 4B, S3). We used three clusters

255 based on the hypothesis that the solutions would group together by the model type (i.e. consensus,

256 consensus without CarveMe, KBase draft models) rather than by the applied approach (i.e. with

257 and without considering the community composition). We found that the reaction set added to the

258 consensus models using COMMIT clearly separated from the other sets of added reactions,

259 indicating that the result from the proposed approach differs substantially from those obtained by

260 gap filling the models individually. The two other clusters were formed by KBase draft models and

261 consensus without CarveMe models including the individual solution for the full consensus,

262 respectively (Figure 4B).

\section{Exchanged metabolites reveal putative metabolic interactions}

265 As the reduction of added reaction sets is based on the metabolic interactions between the models,

266 the underlying set of exchanged metabolites was investigated to find putative dependencies within

267 the investigated communities. To this end, we determined the contribution of each community

268 member to the common pool of exchanged metabolites, without considering those that were

269 already contained in the minimal medium.

270 First, we grouped the models based on bacterial families as found in At-SPHERE (37). As

271 expected, members of all families show putative dependencies for carbohydrates, of which mostly

$272 \beta$-D-galactose but also D-mannitol, maltotriose, maltopentaose, and $\beta$-D-fructofuranose were

273 imported (Figure 5, Bulgarelli: Figure S4). In addition, amino acids, like: D-alanine, L-cysteine, L-

274 leucine, L-asparagine, L-valine, L-isoleucine, L-arginine, and L-lysine were found to be exchanged.

275 Further, we observed that members of the Micrococcaceae appear to have a rather broad spectrum

276 of utilized nutrients, which spans most of the exchanged amino acids and carbohydrates. In

277 contrast, bacterial families, such as: Xanthomonadaceae, Paenibacillaceae, and

278 Mycobacteriaceae, were found to depend on a smaller, more specific set of imported metabolites.

279 Although members of most of the bacterial families can export a variety of metabolites at a relatively

280 low cost, there exist families that include more members exporting these metabolites (e.g.

281 Mycobacteriaceae) than others (e.g. Micrococcaceae). Interestingly, cytidine, L-leucine, L-lysine,

282 iminosuccinate, and iso-citrate were reported to be exchanged between only two families, 
283 respectively, indicating specialized interactions. In contrast, $\mathrm{H}^{+}, \mathrm{P}_{\mathrm{i}}, \mathrm{NH}^{+}, \mathrm{Fe}^{2+}, \mathrm{Fe}^{3+}$, and $\mathrm{SO}_{4}{ }^{2-}$ are

284 exported at least from 20 out of 24 members. However, $\mathrm{H}^{+}$import was observed for only 14

285 community members. This finding indicates that the members of a bacterial community prefer

286 different $\mathrm{pH}$ in their local environment. This also suggests that almost all members have the ability

287 to increase the acidity of the soil without a large growth compromise. Similarly, the import of $\mathrm{P}_{\mathrm{i}}$,

$288 \mathrm{NH}^{+}, \mathrm{Fe}^{2+}, \mathrm{Fe}^{3+}$, and $\mathrm{SO}_{4}^{2-}$ was observed in even fewer instances $(n<3)$.

289 To analyse directed interactions between the community members in more detail, we conducted a

290 downstream analysis of the gap-filled models using the SMETANA tool (10) (implementation from

291 github.com/cdanielmachado/smetana). For the Schlaeppi community, we obtained a high

292 metabolic interaction potential (MIP) of 0.98 , which indicates that the community members can

293 provide most of the essential nutritional components for itself by exchanging metabolites.

294 SMETANA predicted metabolic interactions between 17 out of the 24 community members. To

295 compare the obtained dependencies with our results, we transformed the directed interactions

296 predicted by SMETANA to undirected interactions defined by the pairwise overlap of imported and

297 exported metabolites. We found that about $21 \%$ of all possible interactions were predicted by both

298 methods. Additionally, $22 \%$ of all OTU pairs were predicted to be non-interacting by both methods,

299 summing up to $43 \%$ agreement between SMETANA and COMMIT (Figure S5). However, the

300 intersection of the sets of exchanged metabolites from both methods consisted of only eight

301 metabolites compared to a total of 28 metabolites from COMMIT and 22 from SMETANA; these

302 included, aside from minerals, $\mathrm{D}$-alanine, L-arginine, L-asparagine, L-cysteine, and $\mathrm{NH}^{+}$.

303 To investigate the functional effects of the dependence on the import of metabolites, we

304 investigated whether or not there is a decrease in growth upon blocking the respective uptake

305 reactions for each metabolite. To this end, we calculated the ratio of biomass flux to the optimal

306 growth rate without any constraint on exchange. In addition, we grouped the models based on

307 these ratios using hierarchical clustering (Figure 6, Bulgarelli: Figure S6). The heatmap shows that

308 resulting groups can partly be explained with the phylogeny, with some of the metabolic

309 dependencies being independent of the taxonomic classification. Further, we observed that many

310 OTUs strongly depend on $\beta$-D-galactose, maltotriose, and D-mannitol as carbon sources, while

311 others prefer amino acids, such as: L-asparagine and D-alanine. Interestingly, two members of the

312 genus Paenibacillus (i.e. Soil522 and Soil787) showed a strong reduction in growth when the import

313 of protons or $\mathrm{H}_{2} \mathrm{O}_{2}$ is blocked. In addition, only one model assigned to the genus Rhodanobacter

314 (Soil772) shows a strong reduction across both compositions when sulphur was not available.

315 Indeed, it has been shown that a bacterium of the same genus, Rhodanobacter thiooxydans sp.

316 nov. was found to potentially use sulphur as energy source for autotrophic denitrification (50).

317 Therefore, our findings show that the fully automated metabolic reconstructions while considering 
318 the composition of microbial community and metabolite leakage results in high-confidence models,

319 as corroborated by existing evidence.

Functional separation of the metabolic capabilities in a microbial community represents an important factor directing ecosystem functioning. While ecological interactions describe the effects that species can have on one another they miss out on complex metabolic relationships between multiple species. Leaky functions as metabolite diffusion within microbial communities can more likely explain the underlying metabolic interactions and dependencies (6). The investigation of communities is challenged by the number of participating members and the lack of detailed species-level information.

We investigated the usage of consensus metabolic models for assessing metabolic interactions between the members of a soil community. This was achieved by devising a novel approach, which fills gaps in the metabolic models while considering the community composition. More specifically, the obtained solution is dependent on the permeability-based metabolite exchange between the single models which allows for an investigation of their metabolic dependencies. First, we demonstrated that the draft models generated using KBase (13), CarveMe (14), RAVEN 2.0 (17), and AuReMelPathway Tools (15) differ substantially from each other, as previously indicated by Mendoza et al. (24). We hypothesized that the consensus models generated from the draft models may resemble manually curated reference models of the same species. We showed that, despite exclusively using automated procedures, consensus models are highly organismspecific. Some of the models sharing only the same genus were structurally more similar to the reference than the ones which share the same species. Since in bacteria even strains within a species can differ from each other, the most similar models of the same genus could indeed also be of the same species or be similar due to metabolic niche adaptation. These results suggests that the draft models from different approaches complement each other. Further, pairwise Jaccard distances based on different model features and SVD distance based on the stoichiometric matrices correlated significantly with the phylogenetic distance. These results showed that the

349 The consensus models were then used as input to COMMIT to find a minimal set of reactions 350 allowing growth simulations. As a result, COMMIT both shapes and significantly reduces the gap351 filling solution. However, the set of added reactions by COMMIT was much smaller than that 352 obtained by gap filling of individual models. In addition, we did not observe a significant correlation 353 between the number of exported metabolites per model and the order in which models are 354 considered for gap filling by COMMIT. Therefore, we concluded that the models that are gap-filled 
355 first, in the considered orderings of community members, provide specific compounds to the

356 community that cannot be produced by other members. To corroborate this claim, we did not

357 observe a reduction of biomass flux upon blocking of metabolites unique to the first five models in

358 the optimal gap-filling order.

359 To confirm the putative dependencies, we investigated biomass reduction upon unavailability of

360 each compound in the medium. Here, we observed that the metabolic dependencies of the models

361 clustered in part according to phylogeny. Additionally, auxotrophies appeared to be not only

362 influenced by lineage but also by specific community composition. This is in line with previous

363 observations that auxotrophies can evolve relatively fast, given an environment or community

364 providing the respective compounds, e.g. certain amino acids (51).

365 Finally, we assessed whether the given communities are, as hypothesized, divided into helpers

366 and beneficiaries according to the Black Queen hypothesis (6). Even though the differences in

367 exported metabolites were not large, it could be observed that members of the Mycobacteriaceae

368 are among the OTUs that exported the most metabolites in both communities. Moreover, there

369 were differences between the numbers of imported metabolites between bacterial families, which

370 supports the existence of 'helpers' and 'beneficiaries' within the community.

371 By the community-dependent generation of functional metabolic models, we could show that

372 prediction of metabolic capabilities as well as insight into the community structure can be obtained

373 by solely using automated tools. In conclusion, this study shows that the usage of automatically-

374 generated metabolic models provides a powerful means to analyse large-scale microbial

375 communities even of uncharacterized species.

\section{Materials and Methods}

381 The 432 genomes that were used for genome-scale metabolic model reconstruction were 382 downloaded from At-SPHERE (http://www.at-sphere.com) (37). The phylogeny information as well 383 as the sequences from bacterial culture collections were also obtained from At-SPHERE. 384 Abundances of the single OTUs in the environmental samples were computed using 16S rRNA 385 sequences that were kindly provided by Ruben Garrido Oter (Max-Planck-Institute for plant 386 breeding, Cologne). For this purpose, the USEARCH v11 software (52) was used to generate an 387 OTU table, which was in turn normalized using the $\mathrm{R}$ implementation of the cumulative sum of 388 squares (CSS) method (53). As an intermediate, the BIOM format (54) was used, and conversion 389 was performed by the respective Python and $\mathrm{R}$ implementations.

\section{Draft model generation}


392 The selected approaches, namely: CarveMe (14), KBase (13), RAVEN 2.0 (17), and AuReMe/

393 Pathway Tools $(15,16)$, use different annotation methods and databases which increases the 394 variability of the automatically reconstructed models. For the CarveMe and RAVEN 2.0 draft 395 reconstructions, the structural annotation from At-SPHERE was employed. CarveMe uses a 396 manually curated, universal bacterial model in which BiGG reactions are linked to sequences. Upon 397 sequence comparisons to the provided reference, reactions with low sequence similarity score of 398 their associated enzymes are removed from the universal model. This approach, thus, guarantees 399 an initial functional model given the medium that was used during the reconstruction. Moreover, 400 draft models reconstructed with CarveMe contain a universal biomass reaction, as suggested by 401 Xavier et al. (55).

402 The RAVEN 2.0 reconstruction pipeline for KEGG-based models constructs multiple sequence 403 alignments (MSA) for all KEGG Orthology (KO) sequences, which were filtered for phylogenetically 404 close sequences. Hidden Markov Models (HMM) were computed based on the MSAs and 405 subsequently searched for in the genome using HMMer v3.2.1 (56). In addition, a MetaCyc-based 406 model was generated based on sequence comparison to a complete model of the MetaCyc reaction 407 database. Both model types were created and merged for every OTU using functions of the RAVEN 4082.0 toolbox.

409 To reconstruct draft models using KBase, the genome sequences were re-annotated using RAST 410 (57) with default parameters. To this end, we used the nucleotide sequences of the assemblies. 411 The functional annotation from RAST is linked to reactions in the ModelSEED reaction database, 412 allowing for the reconstruction of a draft metabolic model. The draft models were then downloaded 413 from KBase for further processing.

414 Further, AuReMe/Pathway Tools was used as a fourth reconstruction method. These models were 415 generated based on data files generated by Pathway Tools (16). As a functional annotation is 416 required as an input for the Pathway Tools pipeline, all genomes were annotated using DFAST (58) 417 using default parameters. The resulting draft models from AuReMe contained reactions based on 418 the MetaCyc database.

\section{Model comparison}

421 Metabolic models can be compared structurally using similarity measures that either use the 422 stoichiometric matrix itself of particular features of the model (e.g. reaction or metabolite identifiers).

423 The definitions of all distance measures that were used are described in the Supplementary 424 Methods. First, the distance matrices were combined per OTU by finding a compromise matrix 425 using the STATIS method $(59,60)$. Additionally, the Jaccard distance of gene identifiers was 426 calculated between all four methods and the consensus for each OTU. As a second step, all 
427 resulting gene Jaccard distances and compromise matrices were again joined using STATIS (59,

$42860)$.

429

430 Generation of consensus models

431 As a first step, the draft model from all approaches were converted to have the same number of

432 fields, which in turn share a common format. This is particularly important for reactions, metabolites,

433 genes, and gene-protein-reaction (GPR) rules. Reaction and metabolite identifiers were translated

434 to MNXref identifiers using the MNXref reference files for metabolites and reactions. If a periplasmic 435 compartment was present, all metabolites and reactions were treated as extracellular. Gene 436 identifiers of draft models reconstructed using KBase and AuReMe/ Pathway Tools were translated 437 by BLAST+ (61) search against the respective structural annotation obtained from At-SPHERE. 438 For unification reasons, also biomass and exchange reactions, if present, were removed.

\section{Comparison to reference models}

441 The cross-reference OTUs in At-SPHERE (37) contained isolates with species resolution, among

442 them Bacillus megaterium and Methylobacterium extorquens, for which manually-curated models

443 had been published. The models for B. megaterium WSH-002 (42) and M. extorquens AM1 (41) 444 were downloaded and their features were translated to MNXref name space whenever possible.

445 The comparison was hampered by the lack of cross-references to name spaces other than self446 defined identifiers. True positives were defined as those identifiers of the consensus model that 447 were also present in the respective reference. In contrast, false positives were defined as the 448 difference between the consensus and the reference model. Conversely, the difference between 449 the reference model and the consensus comprised the false negatives. Since the true negatives 450 could not be determined, only sensitivity and precision were calculated as measures to assess the 451 quality of model prediction.

452 The genomes of the two reference models were downloaded from NCBI reference numbers 453 CP003017 and CP001510.1. MAFFT online service (62) was used to generate MSAs of the 16S 454 rRNA sequences of the OTUs and the respective reference, and for NJ/UPGMA phylogeny 455 computation to generate a Newick tree that was converted into a distance matrix using Newick 456 Utilities (63). Both MAFFT and Newick Utilities were used with the default parameter set.

458 Biomass reaction and media

459 After the merging draft reconstructions from the four different approaches, a universal biomass 460 reaction was added to the models. The metabolites and their coefficients in the biomass reaction 461 were adopted from the CarveMe reconstructions (14) as they include the universal biomass 462 reaction suggested by (55). In addition, the initial medium was considered by adding exchange 
463 reactions to the models. The initial medium originates from the intersection of predicted auxotrophic 464 media for the given community obtained from KBase, without organic compounds (except D465 glucose as single carbon source).

\section{Prediction of permeability}

468 To predict metabolite permeability, chemical properties, namely:molecular weight (MW), polar 469 surface area (TPSA), number $\mathrm{H}$-bond donors (HBD), number of $\mathrm{H}$-bond acceptors (HBA), rotatable 470 bonds (RB) and predicted octanol/water partition coefficient (XLogP3), were obtained from 471 PubChem (47) for all metabolites in the MNXref database (Figure S7, S8). This was achieved by 472 translating a unique set of 451219 associated InChl keys to PubChem CIDs, whenever possible 473 (116458 out of 451219: 26\%), so molecular properties for 110266 compounds could be obtained. 474 Since not all entries had an associated XLogP3 value (only 11894: 11\%), the missing values were 475 predicted using k-Nearest-Neighbor (kNN) regression with $k=1$ using MW, TPSA, HBD and RB 476 as predictors (Figure S9) (10-fold cross validation (CV): $R_{a d j}^{2}=0.87 ; R M S E=1.26$ ) (validation 477 set: $\left.R_{a d j}^{2}=0.9 ; R M S E=1.11\right)$. The value for $k$ was determined by comparing the performance of 478 multiple kNN models with $1 \leq k \leq 20$ (Figure S9).

479 The chemical properties were used to classify metabolites into likely permeable and otherwise by 480 applying Lipinski's rule of five and including RB and TPSA $(48,49)$. Precisely, the applied rules 481 were: $\mathrm{HBD} \leq 5, \mathrm{HBA} \leq 10, \mathrm{MW} \leq 500 \mathrm{Da}$, TPSA $\leq 140 \AA \AA, R B \leq 10,-0.4 \leq \mathrm{XLogP} 3 \leq 5.6$.

482 If no properties were available, the respective metabolite was classified as not likely to be 483 permeable. The above-described classifier was applied to all metabolites in the gap-filling 484 database, which is depicted in more detail below. In total, 8354 out of 33547 (24.9\%) metabolites 485 were predicted to be likely permeable. This set of highly-permeable metabolites was enriched with 486 carbohydrates and fatty acids, but also nucleic acids and peptides ( $p<0.001$, ChEBI metabolite 487 ontology (64)). The $p$-values were corrected for multiple testing using the Benjamini-Hochberg 488 procedure (Table S5). Out of 4520 transport reactions, 2766 included at least one highly-permeable 489 metabolite.

\section{COMMIT formulation}

492 To perform gap filling of the generated models we implemented he FastGapFilling algorithm (65) 493 in MATLAB and used specific weights for transport reactions (100), metabolic reactions (non494 transport, 50), changing a reaction's directionality (25), reactions including highly-permeable 495 metabolites (subset of transport reactions, 50), reactions with sequence support (25), allowed 496 uptake reactions for metabolites that are exported by other members (1) and exchange reactions $497\left(10^{5}\right)$. The penalties applied in this study resulted from the comparison of different weights that were 498 altered until satisfying results were obtained for selected models. The upper limit for the biomass 
499 flux was constrained to $2.81 h^{-1}$ as this is the observed growth rate for very fast growing bacteria

500 as Vibrio natriegens (66). The latter was used to avoid the flux through the biomass reaction being

501 pushed to its default limit of 1000 , which would allow a higher number of reactions to be added.

502 The additional constraint on biomass is, however, not propagated to the resulting model, as it is

503 only part of the gap-filling LP.

504 The chemically-balanced part of the MNXref database was used as a gap filling database, including

50525,740 reactions and 33,547 metabolites, after removing reactions according to the criteria

506 described below. To this end, all generic compartments were ignored since a unique translation to

507 either cytosol or extracellular space was not possible. Further, the metabolite ID 'MNXM01' was

508 replaced with 'MNXM1' since both encode $H^{+}$. Additionally, export reactions i.e. reactions that

509 include metabolites associated to the '@BOUNDARY' compartment were excluded from the gap-

510 filling database. Reactions including stoichiometric coefficients above 20 were also removed.

511 To determine weights that quantify sequence similarity, HMMs constructed for all KO files were 512 queried against every genome using HMMer (56). The KO identifiers were matched to MNXref 513 reactions via associated KEGG reactions. An E-value of $10^{-6}$ was used as a cut-off. A lower weight 514 was also assigned to transport reactions that include highly-permeable metabolites as indicated 515 above. To this end, a subset of transport reactions that use highly-permeable metabolites was 516 found in the gap-filling database. Further, one can choose to include sink reactions for cytosolic 517 metabolites in the objective of the gap-filling LP. An additional matrix for sink reactions $R_{\operatorname{sink}}$ is then 518 created for metabolites that are predicted to be highly-permeable or take part in a transport reaction 519 in the model. These sink reactions can also be assigned a specific weight. Since a weighted sum 520 of biomass flux and flux through the added reactions is maximized, only those sink reactions will 521 be added that do not largely decrease the biomass flux. The sink reactions that were found during 522 gap-filling will not be added to the model. Instead, export reactions from the cytosol to extracellular 523 space as well as exchange reactions for the respective extracellular compounds are added to the 524 model.

525 For the conditional gap filling, 100 random orderings of the considered models were inspected 526 during COMMIT. The goal is to identify the best ordering with respect to specified criteria. More 527 specifically, the models are gap-filled following a given ordering. To this end, every iteration starts 528 with a minimal medium, which is augmented by the exportable metabolites of the gap-filled models. 529 The medium for the first model in each iteration comprises metabolites that render the particular 530 model auxotrophic. This is used so as to avoid the gap filling of the first model in the ordering to be 531 unrealistically large in comparison to solutions for subsequent models. The auxotrophy media were 532 obtained from KBase using the corresponding function. To arrive at a minimal medium, only 533 nutrients were considered that were found in all auxotrophic media of the considered organisms, 534 excluding amino acids and carbon sources, except for glucose. After each single gap-filling run, 
535 exportable metabolites are predicted and made available to the subsequent models via additional 536 uptake reactions in the gap-filling database. The prediction of exchanged metabolites can be done 537 during the gap filling step of the individual model or right after. When the first option is used, sink 538 reactions for cytosolic metabolites are integrated directly into the objective of the gap-filling 539 algorithm. Otherwise, a similar LP is solved using the gap-filled model maximizing the flux through 540 sink reactions without reducing the biomass production by more than the given factor. For the 541 second option, the optimal biomass flux $v_{\text {bio }}^{o p t}$ is determined first, followed by the maximization of

542 flux through the sink reactions while guaranteeing a sub-optimal biomass $v_{\text {bio }}^{o p t *}=f \cdot v_{\text {bio }}^{o p t}$. In this 543 study, $f=0.9$ has been used (please see supplementary Methods for more detail).

544 Linear Program for the inclusion of sink reactions after gap filling:

$$
\max \sum_{i \in R_{\text {sink }}} v_{i}
$$

s.t.

$v_{\text {bio }} \geq v_{\text {bio }}^{\text {opt } *}$.

$$
0 \leq v_{i} \leq 1000
$$

Please, refer to the Supplementary Methods for the full specification of the COMMIT procedure.

\section{Acknowledgments}

P.W. and Z.N. would like to thank the Research Focus Group "Evolutionary Systems Biology" of University of Potsdam for funding. Z.N. would like to thank the Max Planck Society for funding.

\section{Author contributions}

Z.N. and P.W. designed the research. P.W. performed all simulations and data analyses. Z.N. and P.W. wrote the manuscript. All authors read and approved the final manuscript.

\section{Competing interests}

The authors declare no competing interest.

\section{References}

1. Emmerson,M.C., Solan,M., Emes,C., Paterson,D.M. and Raffaelli,D. (2001) Consistent patterns and the idiosyncratic effects of biodiversity in marine ecosystems. Nature, 411, 7377.

2. Hooper,D.U., Chapin III,F.S., Ewel,J.J., Hector,A., Inchausti,P., Lavorel,S., Lawton,J.H., Lodge,D.M., Loreau,M., Naeem,S., et al. (2005) Effects of biodiversity on ecosystem functioning: a consensus of current knowledge. Ecol. Monogr., 75, 3-35. 
Molyneaux,P.L., Paredes,R., Pérez Brocal,V., et al. (2017) The microbiome in respiratory medicine: current challenges and future perspectives. Eur. Respir. J., 49, 1602086.

4. Karkman,A., Lehtimäki,J. and Ruokolainen,L. (2017) The ecology of human microbiota: dynamics and diversity in health and disease. Ann. N. Y. Acad. Sci., 1399, 78-92.

5. Pandhal,J. and Noirel,J. (2014) Synthetic microbial ecosystems for biotechnology. Biotechnol. Lett., 36, 1141-1151.

6. Morris,J.J. (2015) Black Queen evolution: the role of leakiness in structuring microbial communities. Trends Genet., 31, 475-482.

7. Yamagishi,J.F., Saito,N. and Kaneko,K. (2020) The advantage of leakage of essential metabolites and resultant symbiosis of diverse species. Phys. Rev. Lett., 124, 048101.

8. Freilich,S., Zarecki,R., Eilam,O., Segal,E.S., Henry,C.S., Kupiec,M., Gophna,U., Sharan,R. and Ruppin,E. (2011) Competitive and cooperative metabolic interactions in bacterial communities. Nat Commun, 2, 589.

9. Mee,M.T., Collins,J.J., Church,G.M. and Wang,H.H. (2014) Syntrophic exchange in synthetic microbial communities. Proc. Natl. Acad. Sci., 111, E2149-E2156.

10. Zelezniak,A., Andrejev,S., Ponomarova,O., Mende,D.R., Bork,P. and Patil,K.R. (2015) Metabolic dependencies drive species co-occurrence in diverse microbial communities. Proc. Natl. Acad. Sci. U. S. A., 112, 6449-6454.

11. Zomorrodi,A.R. and Segrè,D. (2017) Genome-driven evolutionary game theory helps understand the rise of metabolic interdependencies in microbial communities. Nat Commun, 8, 1563.

12. Dillard,L.R., Payne,D.D. and Papin,J.A. (2021) Mechanistic models of microbial community metabolism. Mol. Omi., 10.1039/d0mo00154f.

13. Arkin,A.P., Cottingham,R.W., Henry,C.S., Harris,N.L., Stevens, R.L., Maslov,S., Dehal,P., Ware,D., Perez,F., Canon,S., et al. (2018) KBase: The United States Department of Energy Systems Biology Knowledgebase. Nat. Biotechnol., 36, 566-569.

14. Machado,D., Andrejev,S., Tramontano,M. and Patil,K.R. (2018) Fast automated reconstruction of genome-scale metabolic models for microbial species and communities. Nucleic Acids Res., 46, 7542-7553.

15. Aite,M., Chevallier,M., Frioux,C., Trottier,C., Got,J., Cortés,M.P., Mendoza,S.N., Carrier,G., Dameron,O., Guillaudeux,N., et al. (2018) Traceability, reproducibility and wiki-exploration for "à-la-carte" reconstructions of genome-scale metabolic models. PLOS Comput. Biol., 14, e1006146.

16. Karp,P.D., Latendresse,M., Paley,S.M., Krummenacker,M., Ong,Q.D., Billington,R., Kothari,A., Weaver,D., Lee,T., Subhraveti,P., et al. (2016) Pathway Tools version 19.0 update: software for pathway/genome informatics and systems biology. Brief. Bioinform., 17, 877-890.

17. Wang,H., Marcišauskas,S., Sánchez,B.J., Domenzain,I., Hermansson,D., Agren,R., Nielsen,J. and Kerkhoven,E.J. (2018) RAVEN 2.0: A versatile toolbox for metabolic network 
reconstruction and a case study on Streptomyces coelicolor. PLOS Comput. Biol., 14,

18. Dias,O., Rocha,M., Ferreira,E.C. and Rocha,I. (2015) Reconstructing genome-scale metabolic models with merlin. Nucleic Acids Res., 43, 3899-3910.

19. Henry,C.S., DeJongh,M., Best,A.A., Frybarger,P.M., Linsay,B. and Stevens,R.L. (2010) High-throughput generation, optimization and analysis of genome-scale metabolic models. Nat. Biotechnol., 28, 977-982.

20. Kanehisa,M. and Goto,S. (2000) KEGG: kyoto encyclopedia of genes and genomes. Nucleic Acids Res., 28, 27-30.

21. King,Z.A., Lu,J., Dräger,A., Miller,P., Federowicz,S., Lerman,J.A., Ebrahim,A., Palsson,B.O. and Lewis,N.E. (2016) BiGG Models: A platform for integrating, standardizing and sharing genome-scale models. Nucleic Acids Res., 44, D515-D522.

22. Moretti,S., Martin,O., Van Du Tran,T., Bridge,A., Morgat,A. and Pagni,M. (2016) MetaNetX/MNXref - Reconciliation of metabolites and biochemical reactions to bring together genome-scale metabolic networks. Nucleic Acids Res., 44, D523-D526.

23. Caspi,R., Billington,R., Keseler,I.M., Kothari,A., Krummenacker,M., Midford,P.E., Ong,W.K., Paley,S., Subhraveti,P. and Karp,P.D. (2020) The MetaCyc database of metabolic pathways and enzymes-a 2019 update. Nucleic Acids Res., 48, D455-D453.

24. Mendoza,S.N., Olivier,B.G., Molenaar,D. and Teusink,B. (2019) A Systematic Assessment Of Current Genome-Scale Metabolic Reconstruction Tools. Genome Biol., 20, 158.

25. Chindelevitch,L., Stanley,S., Hung,D., Regev,A. and Berger,B. (2012) MetaMerge: scaling up genome-scale metabolic reconstructions with application to Mycobacterium tuberculosis. Genome Biol., 13, r6.

26. Aung,H.W., Henry,S.A. and Walker,L.P. (2013) Revising the Representation of Fatty Acid, Glycerolipid, and Glycerophospholipid Metabolism in the Consensus Model of Yeast Metabolism. Ind. Biotechnol., 9, 215-228.

27. Lundberg,D.S., Lebeis,S.L., Paredes,S.H., Yourstone,S., Gehring,J., Malfatti,S., Tremblay,J., Engelbrektson,A., Kunin,V., Del Rio,T.G., et al. (2012) Defining the core Arabidopsis thaliana root microbiome. Nature, 488, 86-90.

28. Huttenhower,C., Gevers,D., Knight,R., Abubucker,S., Badger,J.H., Chinwalla,A.T., Creasy,H.H., Earl,A.M., FitzGerald,M.G., Fulton,R.S., et al. (2012) Structure, function and diversity of the healthy human microbiome. Nature, 486, 207-214.

29. Biggs,M.B. and Papin,J.A. (2017) Managing uncertainty in metabolic network structure and improving predictions using EnsembleFBA. PLoS Comput. Biol., 13, e1005413.

30. Klitgord,N. and Segrè,D. (2010) Environments that Induce Synthetic Microbial Ecosystems. PLOS Comput. Biol., 6, e1001002.

31. Pacheco,A.R., Moel,M. and Segrè,D. (2019) Costless metabolic secretions as drivers of interspecies interactions in microbial ecosystems. Nat. Commun., 10, 103. 
32. Popp,D. and Centler,F. (2020) $\mu$ BialSim: Constraint-Based Dynamic Simulation of Complex Microbiomes. Front. Bioeng. Biotechnol., 8, 574.

33. Harcombe,W.R., Riehl,W.J., Dukovski,I., Granger,B.R., Betts,A., Lang,A.H., Bonilla,G., Kar,A., Leiby,N., Mehta,P., et al. (2014) Metabolic Resource Allocation in Individual Microbes Determines Ecosystem Interactions and Spatial Dynamics. Cell Rep., 7, 11041115.

34. Chan,S.H.J., Simons,M.N. and Maranas,C.D. (2017) SteadyCom: Predicting microbial abundances while ensuring community stability. PLOS Comput. Biol., 13, e1005539.

35. Schlaeppi,K., Dombrowski,N., Oter,R.G., van Themaat,E. and Schulze-Lefert,P. (2014) Quantitative divergence of the bacterial root microbiota in Arabidopsis thaliana relatives. Proc. Natl. Acad. Sci., 111, 585-592.

36. Bulgarelli,D., Rott,M., Schlaeppi,K., van Themaat,E., Ahmadinejad,N., Assenza,F., Rauf,P., Huettel,B., Reinhardt,R., Schmelzer,E., et al. (2012) Revealing structure and assembly cues for Arabidopsis root-inhabiting bacterial microbiota. Nature, 488, 91-95.

37. Bai,Y., Müller,D.B., Srinivas,G., Garrido-Oter,R., Potthoff,E., Rott,M., Dombrowski,N., Münch,P.C., Spaepen,S., Remus-Emsermann,M., et al. (2015) Functional overlap of the Arabidopsis leaf and root microbiota. Nature, 528, 364-369.

38. Hamilton,J.J. and Reed,J.L. (2014) Software platforms to facilitate reconstructing genomescale metabolic networks. Environ. Microbiol., 16, 49-59.

39. Mohammadi,R., Fallah-Mehrabadi,J., Bidkhori,G., Zahiri,J., Javad Niroomand,M. and Masoudi-Nejad,A. (2016) A systems biology approach to reconcile metabolic network models with application to Synechocystis sp. PCC 6803 for biofuel production. Mol Biosyst, $12,2552-2561$.

40. van Heck,R.G.A., Ganter,M., dos Santos,V.A.P. and Stelling,J. (2016) Efficient Reconstruction of Predictive Consensus Metabolic Network Models. PLOS Comput. Biol., 12, e1005085.

41. Peyraud,R., Schneider,K., Kiefer,P., Massou,S., Vorholt,J.A. and Portais,J.-C. (2011) Genome-scale reconstruction and system level investigation of the metabolic network of Methylobacterium extorquens AM1. BMC Syst. Biol., 5, 189.

42. Zou,W., Zhou,M., Liu,L. and Chen,J. (2013) Reconstruction and analysis of the industrial strain Bacillus megaterium WSH002 genome-scale in silico metabolic model. J. Biotechnol., 164, 503-509.

43. Satish Kumar,V., Dasika,M.S. and Maranas,C.D. (2007) Optimization based automated curation of metabolic reconstructions. BMC Bioinformatics, 8, 1-16.

44. Thiele, Ines; Palsson,B., Thiele,I. and Palsson,B.O. (2010) A protocol for generating a highquality genome-scale metabolic reconstruction. Nat. Protoc., 5, 93-121.

45. Bell,T., Newman,J.A., Silverman,B.W., Turner,S.L. and Lilley,A.K. (2005) The contribution of species richness and composition to bacterial services. Nature, 436, 1157-1160.

46. Liu,L., Zhang,Z., Sheng,T. and Chen,M. (2016) DEF: an automated dead-end filling 
approach based on quasi-endosymbiosis. Bioinformatics, 33, 405-413.

692

693

694

695

696

697

698

699

700

701

702

703

704

705

706

707

708

709

710

711

712

713

714

715

716

717

718

719

720

721

722

723

724

725

726

727

728

47. Kim,S., Chen,J., Cheng,T., Gindulyte,A., He,J., He,S., Li,Q., Shoemaker,B.A., Thiessen,P.A., Yu,B., et al. (2019) PubChem 2019 update: Improved access to chemical data. Nucleic Acids Res., 47, D1102-D1109.

48. Lipinski,C.A., Lombardo,F., Dominy,B.W. and Feeney,P.J. (2001) Experimental and computational approaches to estimate solubility and permeability in drug discovery and development settings. Adv. Drug Deliv. Rev., 46, 3-26.

49. Veber,D.F., Johnson,S.R., Cheng,H.Y., Smith,B.R., Ward,K.W. and Kopple,K.D. (2002) Molecular properties that influence the oral bioavailability of drug candidates. J. Med. Chem., 45, 2615-2623.

50. Lee,C.S., Kim,K.K., Aslam,Z. and Lee,S.T. (2007) Rhodanobacter thiooxydans sp. nov., isolated from a biofilm on sulfur particles used in an autotrophic denitrification process. Int. J. Syst. Evol. Microbiol., 57, 1775-1779.

51. D'Souza,G. and Kost,C. (2016) Experimental Evolution of Metabolic Dependency in Bacteria. PLoS Genet., 12, e1006364.

52. Edgar,R.C. (2010) Search and clustering orders of magnitude faster than BLAST. Bioinformatics, 26, 2460-2461.

53. Paulson,J.N., Stine,O.C., Bravo,H.C. and Pop,M. (2013) Differential abundance analysis for microbial marker-gene surveys. Nat. Methods, 10, 1200-1202.

54. McDonald,D., Clemente,J.C., Kuczynski,J., Rideout,J.R., Stombaugh,J., Wendel,D., Wilke,A., Huse,S., Hufnagle,J., Meyer,F., et al. (2012) The Biological Observation Matrix (BIOM) format or: how I learned to stop worrying and love the ome-ome. Gigascience, 1.

55. Xavier,J.C., Patil,K.R. and Rocha,I. (2017) Integration of Biomass Formulations of GenomeScale Metabolic Models with Experimental Data Reveals Universally Essential Cofactors in Prokaryotes. Metab. Eng., 39, 200-208.

56. Eddy,S.R. (1998) Profile hidden Markov models. Bioinformatics, 14, 755-763.

57. Brettin,T., Davis,J.J., Disz,T., Edwards,R.A., Gerdes,S., Olsen,G.J., Olson,R., Overbeek,R., Parrello,B., Pusch,G.D., et al. (2015) RASTtk: a modular and extensible implementation of the RAST algorithm for building custom annotation pipelines and annotating batches of genomes. Sci Rep, 5, 8365.

58. Tanizawa,Y., Fujisawa,T. and Nakamura,Y. (2018) DFAST: a flexible prokaryotic genome annotation pipeline for faster genome publication. Bioinformatics, 34, 1037-1039.

59. Escoufier,Y. (1980) L'analyse conjointe de plusieurs matrices de données. In Legay,J.-M., Tomassone,R., Jolivet,E. (eds), Biométrie et Temps. Société Française de Biométrie, Paris, pp. 59-76.

60. L'Hermier des Plantes,H. (1976) Structuration des tableaux à trois indices de la statistique. Théorie et application d'une méthode d'analyse conjointe Université des Sciences et Techniques du Languedoc, Montpellier. 
61. Camacho,C., Coulouris,G., Avagyan,V., Ma,N., Papadopoulos,J., Bealer,K. and Madden,T.L. (2009) BLAST+: architecture and applications. BMC Bioinformatics, 10, 421.

62. Katoh,K., Rozewicki,J. and Yamada,K.D. (2019) MAFFT online service: multiple sequence alignment, interactive sequence choice and visualization. Brief. Bioinforma., 20, 1160-1166.

63. Junier,T. and Zdobnov,E.M. (2010) The Newick utilities: high-throughput phylogenetic tree processing in the UNIX shell. Bioinformatics, 26, 1669-1670.

64. Hastings,J., Owen,G., Dekker,A., Ennis,M., Kale,N., Muthukrishnan,V., Turner,S., Swainston,N., Mendes,P. and Steinbeck,C. (2016) ChEBI in 2016: Improved services and an expanding collection of metabolites. Nucleic Acids Res., 44, D1214-D1219.

65. Latendresse,M. (2014) Efficiently gap-filling reaction networks. BMC Bioinformatics, 15, 225.

66. Lee,H.H., Ostrov,N., Wong,B.G., Gold,M.A., Khalil,A.S. and Church,G.M. (2019) Functional genomics of the rapidly replicating bacterium Vibrio natriegens by CRISPRi. Nat. Microbiol., 4, 1105-1113. 


\section{Figures and Tables}

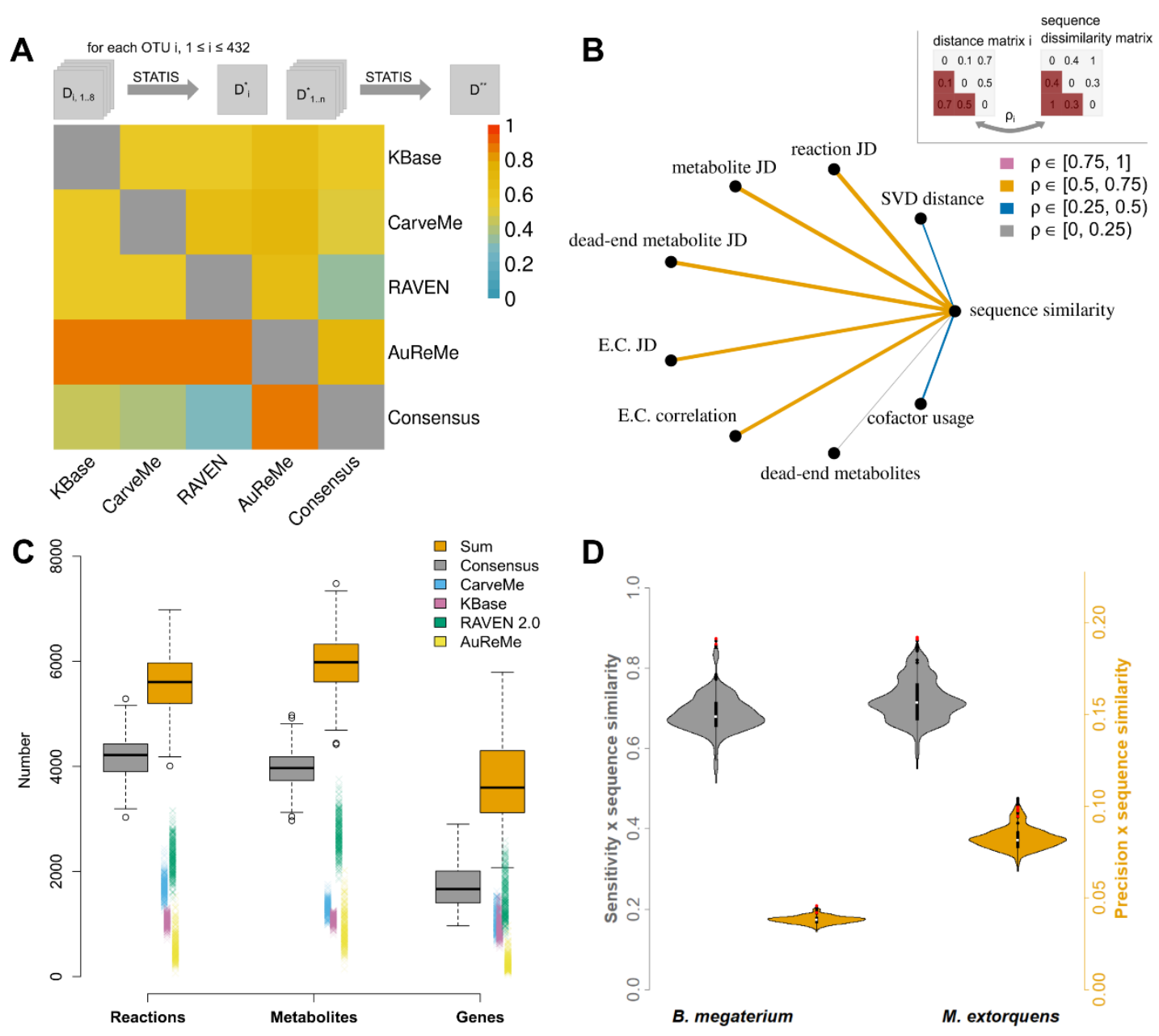

Figure 1. Structural comparisons of draft and consensus models. (A) Structural differences across the reconstructed draft models and the consensus. upper triangle: For each OTU, eight distance matrices $(D)$ were computed to compare the models reconstructed using KBase (13),

748 CarveMe (14), RAVEN 2.0 (17), AuReMe/ Pathway Tools (15, 16), and the consensus model.

749 Detailed information on the distance measures can be found in the Methods section. These 750 matrices were then combined per OTU by finding a compromise matrix $D_{i}^{*}$ (STATIS method (59, 751 60)). The resulting matrices (one for each OTU, $n=432$ ) were again combined using STATIS, 752 resulting in an approach-by-approach distance matrix $D^{* *}$. lower triangle: Gene Jaccard distances 753 across all four methods and the consensus model. The compromise distance matrices were 754 obtained as described above. Values close to zero indicate smaller differences. (B) Correlation of 755 structural distances between the consensus models with sequence dissimilarity of $16 \mathrm{~S}$ rRNA 756 sequences. The same eight distance measures from (A) were used for pairwise comparisons of 757 consensus models between all OTUs $(n=432)$. Detailed information on the distance measures 
758 can be found in the Methods section. Mantel correlation was used to compare each distance matrix

759 to the sequence dissimilarity as depicted in the top-right corner. (C) Comparison of consensus

760 models to draft models. The numbers of reactions, metabolites, and genes are shown for the

761 consensus model in comparison to the single draft models generated by the four approaches. To

762 depict the overlap between the four approaches, the sum of numbers of reactions, metabolites, and

763 genes per model is shown ("Sum", orange box). (D) Similarity of consensus models to selected

764 reference models. The sensitivity (grey, left) and precision (orange, right) with respect to reaction,

765 metabolite, E.C. number sets were calculated for each of the 432 models and each reference

766 model. These values were scaled by the sequence similarity to the genome of the used references.

767 OTUs that were assigned the same genus (9 for B. Megaterium and 27 for $M$. extorquens) or

768 species (2 for B. Megaterium and 5 for M. extorquens) according to Bai et al. (37), are shown as

769 black or red dots.

770 
A

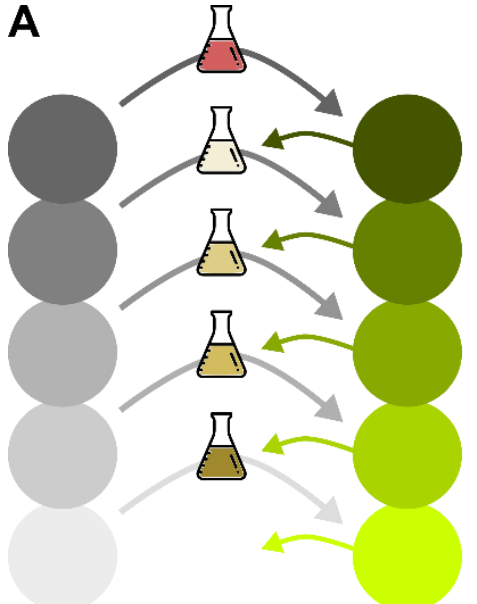

B
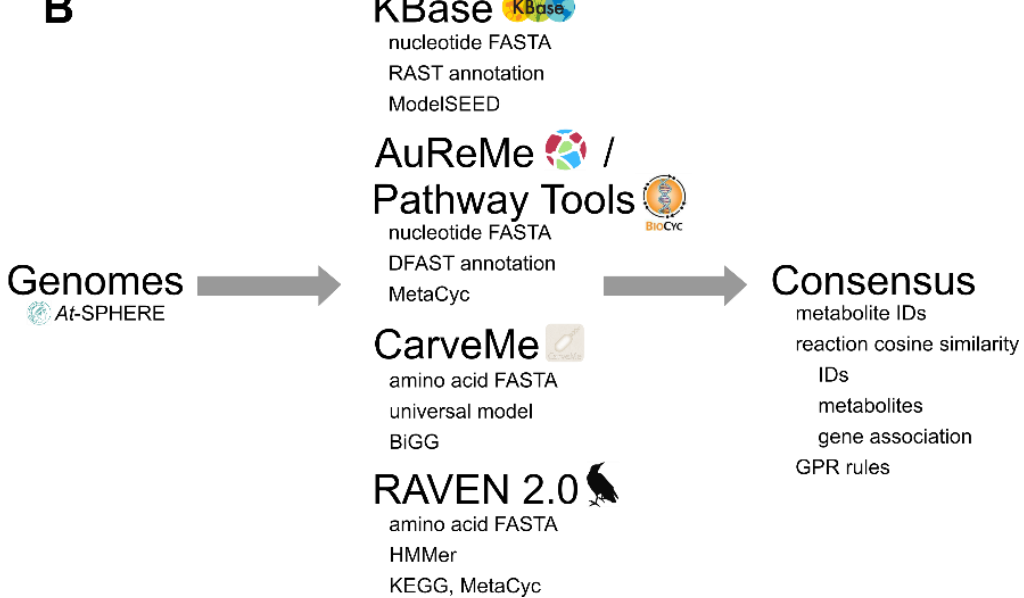

772 Figure 2. Schematic workflow of metabolic model reconstruction from multiple approaches

773 using COMMIT. (A) The consensus models were gap-filled conditional on the community

774 composition. Grey circles represent the models for every organism before gap filling. Green color

775 indicates functional models after gap filling. The medium for the first model was the respective

776 auxotrophic medium predicted using KBase (13) (red medium). For subsequent models, a minimal

777 medium was used, which was enriched by the exported metabolites of already gap-filled models

778 (green arrows). (B) Annotated genomes from At-SPHERE were used as the basis to reconstruct 779432 draft metabolic models using the four recent methods from KBase (13), CarveMe (14), RAVEN

7802.0 (17), and AuReMe/Pathway Tools $(15,16)$. These draft models were merged into consensus 781 models per OTU. 

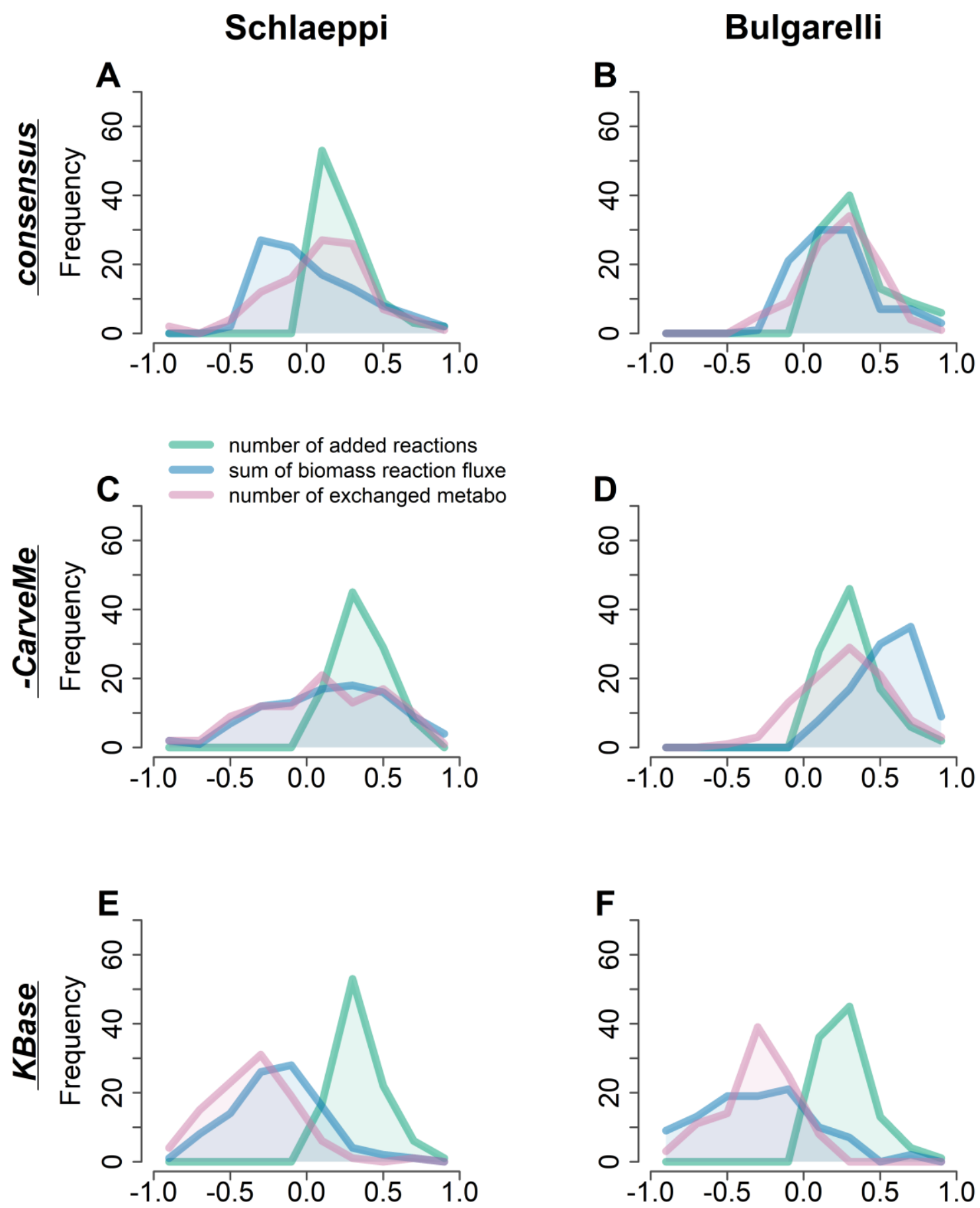

scaled difference to optimal solution

Figure 3. Differences across the random orderings of microorganisms explored in the gap

of biomass fluxes, and the number of exchanged metabolites were compared between all orderings explored by COMMIT. (A,B) consensus models from all approaches (C,D) consensus models without CarveMe draft models (E,F) converted KBase draft models. Results from the Schlaeppi 
bioRxiv preprint doi: https://doi.org/10.1101/2021.06.02.446851; this version posted June 3, 2021. The copyright holder for this preprint (which

was not certified by peer review) is the author/funder, who has granted bioRxiv a license to display the preprint in perpetuity. It is made available under aCC-BY-NC 4.0 International license.

789 community are shown on the left and results from the Bulgarelli community on the right panels. The 790 lines represent the number of counts for each histogram bin, for the added reactions, growth rates, 791 and numbers of exchanged metabolites. The values for each measure were scaled to the 792 respective value associated with the optimal ordering. Number of iterations: $n=100$; Number of 793 OTUs per community: 24 for Schlaeppi and 20 for Bulgarelli.

794 
A

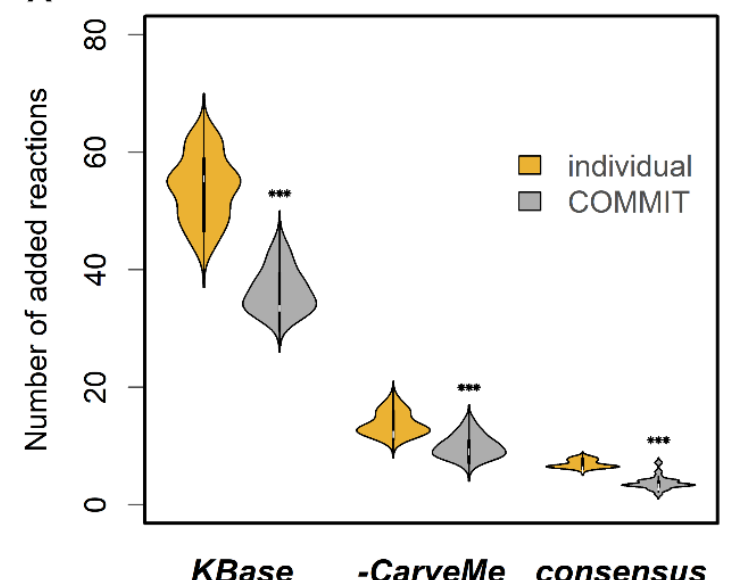

B

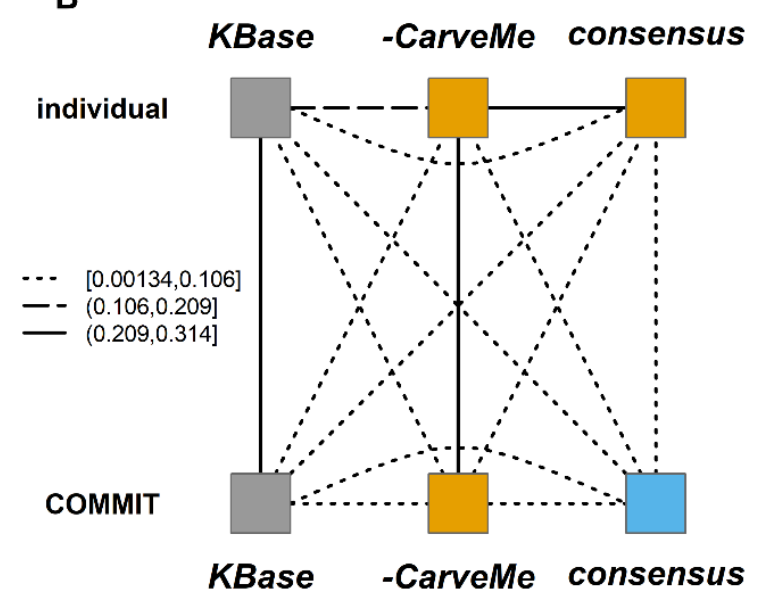
consensus metabolic models in the Schlaeppi community. Full consensus (consensus),

798 consensus without CarveMe models (-CarveMe), and KBase draft models (KBase) were gap-filled 799 either individually or conditionally using the COMMIT approach. (A) Sizes of gap-filling solution sets 800 were compared for each model type using a paired Wilcoxon rank sum test $(* * * p<0.001)$. (B)

801 Pairwise comparison of added reactions obtained for each model and gap-filling type by calculating 802 the Jaccard similarity per OTU. The resulting matrices were merged per group using STATIS 803 method $(59,60)$. The obtained values were grouped using $\mathrm{K}$-means clustering $(K=3)$. The line 804 types indicate the average similarity between the compared groups.

805 


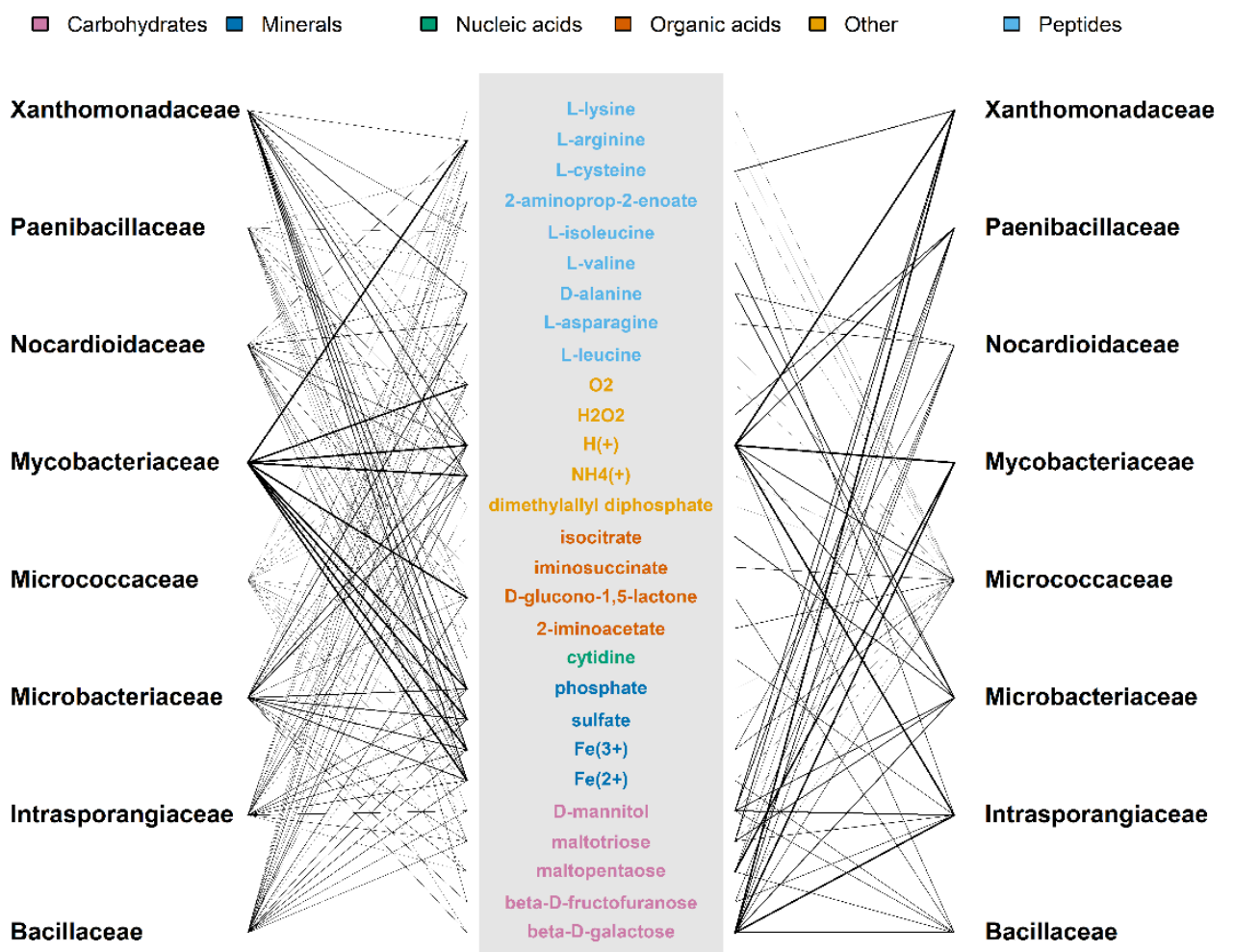

806

Figure 5. Putative metabolic interactions between the bacterial families. The sets of imported and exported metabolites that were determined for each member during conditional gap-filling and grouped into corresponding bacterial families. On the left-hand side, metabolite export is shown, which does not require a reduction of growth greater than $10 \%$. The common pool of exchangeable metabolites is shown in the center, which were classified using the KEGG BRITE br08001 with manual refinement. On the right-hand side, import reactions are shown, which were introduced during the gap-filling procedure. Line widths represent the number of community members per 815 


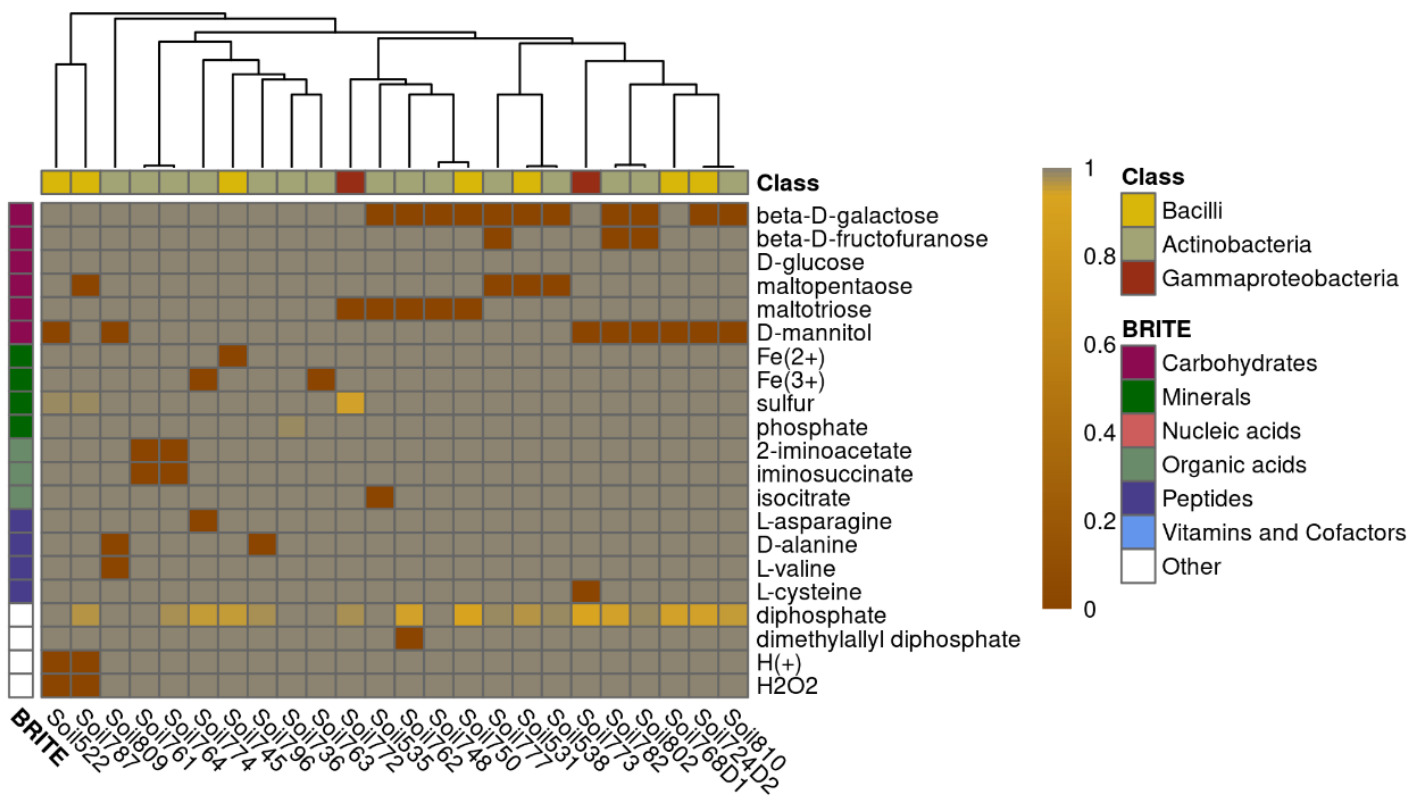

817 Figure 6. Reduction of biomass flux upon blocking the uptake of metabolites whose import

818 was added during conditional gap filling. The uptake of every metabolite whose uptake was

819 included during conditional gap filling was blocked separately and the growth rate under this

820 condition was divided by the growth rate for that particular model. A threshold of 0.99 was applied

821 to avoid false-positive responses. Biomass flux ratios were calculated for all metabolic models in

822 the Schlaeppi community. The models were annotated to classes based on the taxonomy published

823 by Bai et al. (37). The metabolites were grouped based on KEGG BRITE br08001, which was

824 manually refined. Hierarchical clustering was performed using average linkage with Euclidean

825 distance. Cells in dark red indicate low biomass whereas yellow cells indicate a smaller reduction

826 of biomass. 